\title{
LiDAR digital building models for true orthophoto generation
}

\author{
Luigi Barazzetti • Maria Antonia Brovelli • \\ Luana Valentini
}

Received: 25 September 2009 / Accepted: 30 September 2010 /Published online: 15 October 2010

(C) The Author(s) 2010. This article is published with open access at Springerlink.com

\begin{abstract}
The importance of digital orthophotos in spatial databases has increased in recent years, since they are an efficient, low-cost and, if properly managed, accurate product. Usually, the generation of orthophotos is carried out using digital terrain models (DTMs); meaning without taking into account vegetation, buildings, and other attached and detached structures. This leads to low accuracies in urban areas, bringing distortions into the image. To avoid this unwanted effect, one must adopt a digital surface model (DSM), as proposed by Amhar et al. (Int Arch Photogrammetry Remote Sens 32(4):16-22, 1998). The method proposed in this paper allows for the creation of true orthophotos by using a DSM to refine the representation of buildings. The pixel size of the DSM must be similar to that of the true orthophoto in order to model the roof edges with sufficient accuracy. This paper presents a new method capable of correcting the roof displacement using an approach based on the integration of several products today available in public administrations, such as a geodatabase, DTMs/DSMs, and light detection and ranging (LiDAR) data. The method is based on a rigorous modelling of simple roofs starting from their 2D projection in the geodatabase, while information about their heights can be obtained using LiDAR data. For some selected simple roofs, automatic modelling can be carried out, in which a robust interpolation method, such as RANSAC, is
\end{abstract}

\footnotetext{
L. Barazzetti $\cdot$ M. A. Brovelli $\cdot$ L. Valentini $(\triangle)$

Politecnico di Milano, DIIAR,

Piazza L. da Vinci 32,

20133 Milan, Italy

e-mail: luana.valentini@mail.polimi.it

L. Barazzetti

e-mail: luigi.barazzetti@polimi.it

M. A. Brovelli

e-mail: maria.brovelli@polimi.it
}

used to model the pitches identified by a clustering procedure. For complex roofs, where creating a rigorous model in a fully automatic way is not possible, a procedure based on the thickening of a DSM is carried out.

Keywords LiDAR - True orthophoto - Digital surface model $\cdot$ Modelling $\cdot$ Building reconstruction

\section{Introduction}

Thanks to their low cost, nowadays, aerial orthophotos are an often requested product, especially for public administrations. Considering the nominal scale of orthophotos $(1: 2,000)$, avoiding errors greater than few decimetres is necessary.

The quality of orthophotos depends on many factors, such as image resolution, accuracy of the camera calibration and orientation, and digital terrain model (DTM) accuracy. In particular, to produce an orthophoto at a defined nominal scale, one must use a set of data with coherent accuracies to practical standards. Additionally, using a DTM, only the height of the bare terrain is considered, and, therefore, ordinary orthophotos show significant positioning errors. On the other hand, using a digital surface model (DSM), this problem is solved, and true orthophotos are produced.

The positioning error due to the deformation introduced through the use of the DTM is directly proportional to the distance between the measured point and the nadir, to the vertical offset of the object with respect to the DTM, and it is inversely proportional to the principal distance of the camera (Kraus 2007). This aspect should be carefully considered in urban landscapes with high buildings and vegetation.

In the past, dense DTMs/DSMs were generated at low cost using only aerial photogrammetry. However, now, we 
can use light detection and ranging (LiDAR) technology to acquire $3 \mathrm{D}$ data and derive high-resolution models.

The resulting orthophotos do not have perspective deformations, and overlapping them with existing geodatabase or vector maps should be possible.

The work described in this paper is a refinement of the method proposed by Barazzetti et al. (2007), hereafter called the "thickening method", based on thickening a LiDAR DSM from an initial grid of $2 \times 2 \mathrm{~m}$ to one of $0.2 \times 0.2 \mathrm{~m}$ spacing.

The generation of accurate orthophotos, based on the use of a DSM to model the surface of the ground and 3D objects on it, was first proposed by Amhar et al. (1998) with the term "true orthophoto". Adopting this procedure, no information is obtained about the ground level area when a building is correctly represented, due to the occlusion produced by the building itself. Moreover, if the generation of orthophotos is based on single images, empty areas cannot be filled; if these areas are modelled using only a DTM, the image content is duplicated. The solution to this problem is the combination of several images from different points of view (Rau et al. 2002; Biason et al. 2003) so that if there is an occlusion area on an image, information about the area can be extracted from other images.

The existing literature proposes several solutions for creating true orthophotos. These solutions involve a multistep procedure. First, one must determine the occluded areas for each single image. This can be done by considering the ray from the perspective centre of the image to the DSM. Occluded areas must be left empty during the rectification process in order to avoid a "ghost effect" (Braun 2003). Then, each rectified image can be used in the mosaic generation process, which fills up occluded areas.

Several approaches for true orthophoto production can be found in the literature. They use different data (e.g., dense DSMs, TIN, geodatabase, and 3D city models). Some examples can be found in Schickler (1998), Dequal and Lingua (2004), Kuzmin et al. (2004), Zhou (2005), Ulm and Poli (2006), and Habib et al. (2007), where manual, semi-automatic, and fully automatic methods are presented.

However, the modelling of man-made objects must be carried out not only by considering their perimeters but also by using a model that takes into account the vertical position of the elements. Moreover, elements that lie on the roof should be modelled (e.g., domes).

All these considerations make true orthophoto production a more complicated task than traditional orthorectification. In fact, the presented issues and other economic aspects should be addressed. Additionally, the availability of more images than those used for traditional rectification, a better model of the object, manual operations, and more complicated algorithms for image mosaicking should be carefully taken into account. Due to higher costs, today true orthophoto production is carried out only in areas of primary importance in presence of tall buildings.

Once the 3D data are available, to generate a proper model of buildings, one must develop automatic data processing algorithms in order to avoid time-consuming interactive editing. Among the numerous automatic reconstruction methods available, techniques allowing for the detection of $3 \mathrm{D}$ building roof planes play an important role. Using the information derived from data, one can detect buildings and create models that correctly represent the roof structures.

The first step is the automatic separation of raw data into three main classes: bare terrain, vegetation, and buildings. The subregions are usually obtained using cluster analysis or region growing. After the classification, one can obtain a geometric reconstruction of the buildings and identify the roof slopes and eaves by means of different methodologies.

For the extraction of buildings, two different approaches are generally used: data-driven and model-driven techniques, which can be integrated with existing knowledge.

The data-driven models try to model each part of the building point cloud in order to obtain the most faithful (or the nearest) polyhedral model (Rottensteiner 2003). The detection of planes can be carried out using many methods, such as region growing, RANdom SAmple Consensus (RANSAC; Fischler and Bolles 1981), or the Hough transform. Once the edges are extracted, they can be grouped into rectangles and then filtered using spectral signature and size. The data-driven generation of correct polyhedral building models is possible only if the data density is high enough to locate a sufficient number of points at least in the most relevant planes of the roof models.

On the other hand, model-driven approaches search for the most appropriate model among primitive building types contained in a database (Maas and Vosselman 1999). After the instantiation of parametric primitives, if sufficient evidence is found, one must fit them to the data.

The use of existing 2D ground plans can help to solve detection problems, select the model in a model-driven approach, or determine the correct position of boundaries and orientations. Problems might be encountered due to inaccurate or outdated maps or with structures for which no hint appears in the ground plan.

Many authors have tried different approaches for the generation of 3D building models based on laser scanner data, using them exclusively or supported by other existing data, such as aerial images or 2D plans.

Geibel and Stilla (2000) applied a region-growing algorithm to LiDAR data, grouping the neighbouring segments, which involved finding consistent intersections at the building vertices. They combined the $3 \mathrm{D}$ border polygons to obtain consistent building models, adding building outlines, vertical walls, and a floor to the model. After generating the building model, they projected its roof edges back to aerial 
images where the model edges were matched with image edges. This technique increased the accuracy of the model, especially with respect to the building outlines.

Brenner (2000) used a bottom-up approach: planar faces were extracted from a regularised DSM using RANSAC and then accepted or rejected based on a set of rules expressing possible relationships between faces and ground plan edges. The final topology of the roof was obtained from all accepted regions through a global search procedure, which uses discrete relaxation and a constrained tree search to cut down search space. To enforce regularity, one must introduce additional constraints and a least squares adjustment.

The method proposed by Vosselman (1999) and refined with Dijkman (Vosselman and Dijkman 2001) extracts faces from non-regularised laser scan data using a Hough transform, followed by connected component analysis. Edges are found through the intersection of faces and analysis of height discontinuities, without using ground plans as additional information. The roof topology is built by bridging gaps in the detected edges; to enforce building regularities, the use of geometric constraints is proposed.

Rottensteiner (2003) presented a method for automatically creating polyhedral building models without using ground plans. From a point cloud characterised by a point distance of $0.1 \mathrm{~m}$ (in-flight) and $0.9 \mathrm{~m}$ (cross-flight), a regular grid for building extraction was derived.

Forlani et al. (2005) computed geometric and topological relationships among regions. In the last step, a rule-based scheme for the classification of the regions was applied, and, then, polyhedral building models were reconstructed analysing the topology of buildings' outlines, roof slopes, and eaves. A similar approach, based on the concept of a roof topology graph to represent the relationships between the various planar patches of a complex roof structure, was followed by Verma et al. (2006).

Recently, a potential-based approach was used iteratively with the k-means algorithm by Sampath and Shan (2008). They adopted clustering techniques to create a polyhedral model of building roofs.

In the case handled by Barazzetti et al. (2007), information about the location of roof borders might be lacking since the DSM grid spacing was higher than the resolution of the orthophoto. Here, the orthorectification algorithm had no information about pixel position, so establishing whether a pixel represented a piece of terrain or roof was not possible. The proposed solution was based on the creation of a LiDAR DSM integrated with a geodatabase. This provided information about the buildings' ground coverage and improved the quality of spatial data, which can be used by institutions devoted to land management and planning.

In this paper, we try to identify different roof slopes exploiting information derived from data analysis, such as aspect values at the point position. Once the slopes are individuated, RANSAC is used to interpolate the data and reconstruct the planes on a regular grid. This method allows for the creation of dense DSMs with a grid spacing similar to that of the true orthophoto, in which roof edges are also correctly modelled. The proposed aspect-based point classification procedure is a fast solution with a reduced computational cost. Moreover, the method can handle different datasets with a variable density of LiDAR points, without increasing the computational cost significantly.

However, this method was developed to generate true orthophotos by means of a grid model of the buildings. Thus, the quality of the final true orthophoto is the goal of primary importance, while the method also gives a $3 \mathrm{D}$ model of the buildings.

The correctness of the model has been tested by orthorectifying a selected area of the municipality of Lecco and comparing the $2 \mathrm{D}$ plans with the true orthophoto obtained with the model itself. The true orthorectification procedure used forces the roofs into the position defined by the geodatabase; therefore, the spatial accuracy is equal to that of the geodatabase. Consequently, a visual inspection is sufficient to check the quality of the final image, in particular close to breaklines where the ribbon-shape effect plays a fundamental role. Several experiments provided sharp roof edges and confirmed the efficiency of this procedure.

\section{Data description}

The LiDAR data used in this work were acquired by Compagnia Generale Riprese Aeree (a BLOM ASA company) in 2005 using the Optech ALTM3033 altimeter. The theoretical density of the points was $0.2-1$ points $/ \mathrm{m}^{2}$ (without taking into account the overlapping area), while the real density in the considered area is about 1.7 points $/ \mathrm{m}^{2}$, which is not sufficient to model small objects like chimneys or antennas. In order to build roof models, only first pulse data are used. Point classification (terrain or building) has been derived from the 1:2,000 geodatabase of the area of interest (an industrial area in Lecco, Italy), which represents building perimeters exactly at the same time of the images. In fact, a geodatabase usually available at an urban scale does not contain the description of single roof slopes but only the building edges.

With the same LiDAR survey, a DTM/DSM featuring a grid of $2 \times 2 \mathrm{~m}$ spacing has been created. These products were used to check the results of our method for true orthophoto production.

The aerial images used were captured in 2003 using an analogue camera RC30/ASCOT equipped with a wideangle lens $(150 \mathrm{~mm})$. The area was covered by several flights, characterised by different heights. In this work, nine images were used. The scale number of the images varies from 4,600 up to 17,000 . Considering a mean scale number 


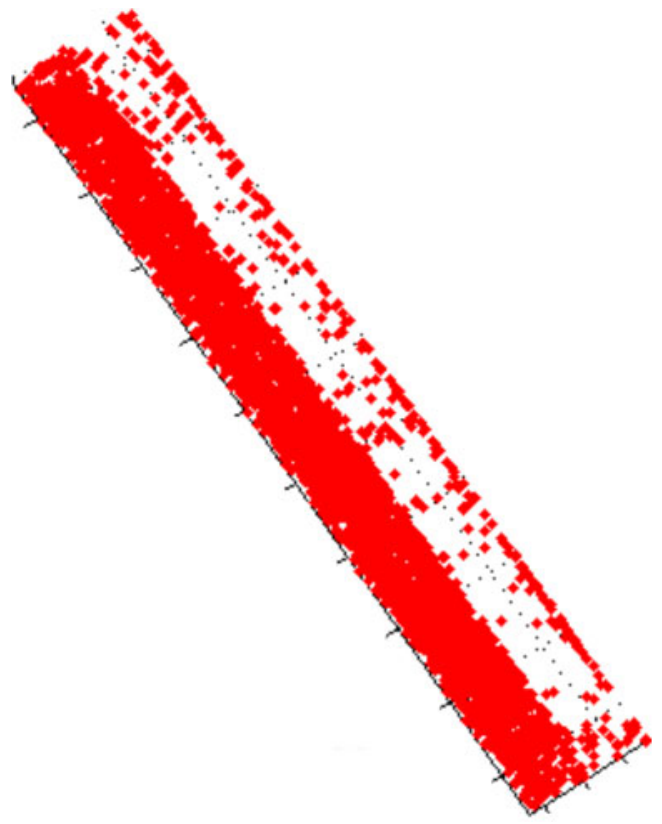

Fig. 1 Example of an aspect class for a simple two slope roof; spread and edge points are clearly visible

for the higher flight equal to 13,000 and the sensor pixel size (equal to $14 \mu \mathrm{m}$ ), we obtain a ground sample distance almost equal to $0.2 \mathrm{~m}$.

\section{Pre-processing steps and aspect computation for point classification}

\section{Data pre-processing}

In order to detect roof points, some pre-processing steps need to be completed. First, using the geodatabase (in

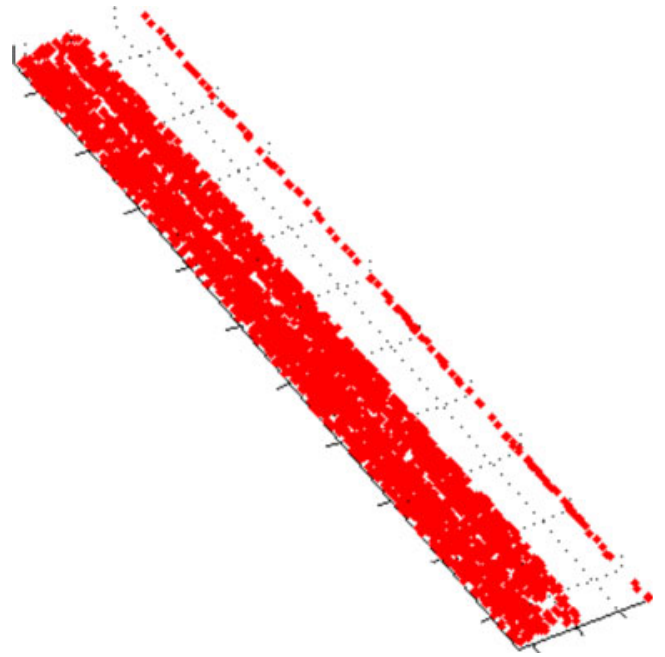

Fig. 2 Example of the previous aspect class (Fig. 1) after the removal of spread points

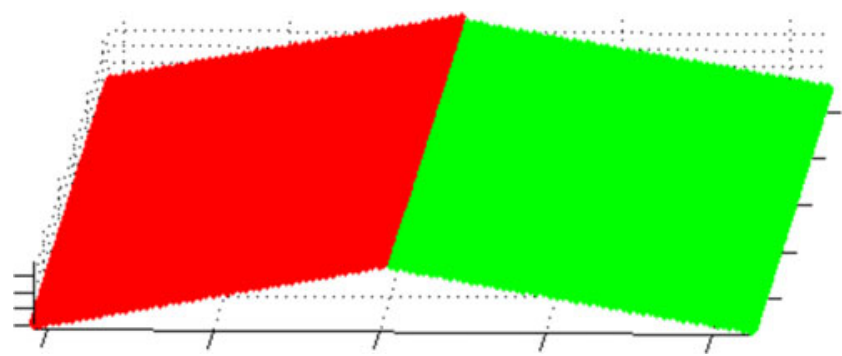

Fig. 3 A model of a simple gabled roof

particular a 2D map of the buildings), a code number is assigned to each point according to the individual building to which it belongs. The points without any code are not taken into account in the following steps.

Both roof and facade points are included in the labelled points; thus, further passes must be carried out to select only those belonging to the roofs. Assuming that facade points are scattered and less frequent, we removed them setting an empirical threshold on the height histogram. For each building, we divided the points into ten equally spaced bins and set the threshold on the height histogram equal to $5 \%$ of the total count of building points. We tested the effectiveness of this selection on several buildings characterised by different dimensions and morphological features. For $77 \%$ of the cases, the error committed was less than 5\%. For $20 \%$, it was between $5 \%$ and $10 \%$, and, for $3 \%$, it was over $10 \%$. These errors were mainly caused by the presence of some points lying on the terrain at the base of the buildings and can be removed exploiting the DTM information. Interpolating the values of the DTM with bicubic splines and adopting relative height values, we can easily remove points at a height less than $3 \mathrm{~m}$ (assuming that the minimum height of a building cannot be less than $3 \mathrm{~m}$ ). In this way, we can sensibly improve data quality, reducing errors to below $1 \%$.

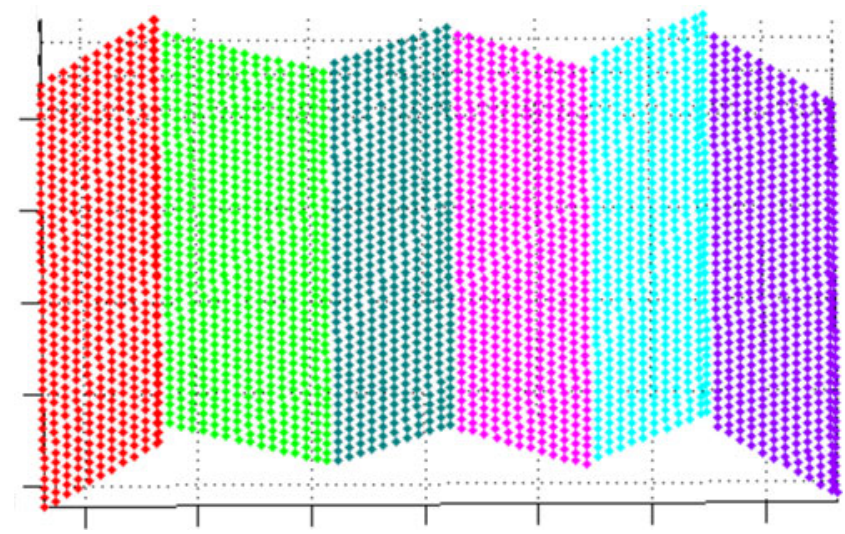

Fig. 4 A model of a roof with several pitches 


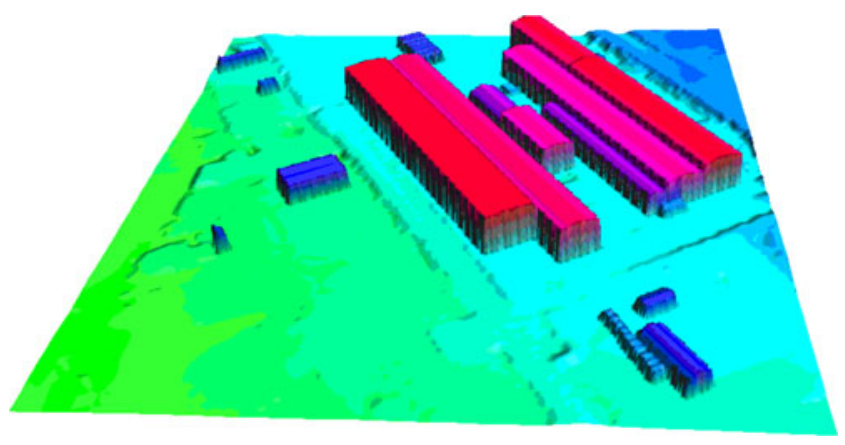

Fig. 5 The grid model of the considered buildings

\section{Aspect computation}

To perform data classification, aspect values were taken into account. Aspect is defined as the azimuth of the steepest down-slope direction of the surface at the considered point (De Smith et al. 2007) and is given in degrees (from $0^{\circ}$ to $360^{\circ}$ ) with respect to the north direction. It is usually divided into four classes: north (from $0^{\circ}$ to $45^{\circ}$ and from $315^{\circ}$ to $360^{\circ}$ ), east (from $45^{\circ}$ to $135^{\circ}$ ), south (from $135^{\circ}$ to $225^{\circ}$ ), and west (from $225^{\circ}$ to $315^{\circ}$ ).

As observations are affected by errors, the estimation of the derivatives increases errors. This leads to a poor solution, in which points can be classified in all the four classes even if the roof has just one slope. As a consequence, subdivision into the different roof types is hard to automate. For simplicity, we considered only a few roof types in this phase of the work:

- $\quad$ tilted roofs,

- flat roofs,

- gabled roofs.
However, to obtain a complete model of the buildings in the considered area, we used the "thickening method" so that we could also model complex roofs in an automatic way (see the 'Model reconstruction on a regular grid' section).

Roofs with just one slope are characterised by having an aspect class with a percentage higher than 50. For flat roofs, the percentages of aspect classes are not relevant, but looking at the point heights is useful: If the difference between the $75^{\circ}$ and the $25^{\circ}$ percentile is smaller than $0.5 \mathrm{~m}$, we can suppose that the roof is flat. The value $0.5 \mathrm{~m}$ was checked empirically.

\section{Data enhancing for roofs with several pitches}

This type of roof (with several pitches) requires further removal of some scattered points in order to perform a good planar interpolation and divide the points in the different roof planes, especially industrial roofs with several pitches. Moreover, we have to separate the points lying on the slopes from those belonging to the opposite roof edges that are wrongly classified as of the same aspect class (see Fig. 1).

The implemented algorithm works along the direction of maximum variation that represents the direction along which we can divide points in different clusters and creates the histogram for the selected coordinate ( $x$ or $y$ according to the chosen direction). Setting an empirical threshold equal to $20 \%$ of the points belonging to the biggest bar of the histogram, points that fall on bars with less elements are removed. In this way, we can eliminate spread points, as is visible in Fig. 2.

After this step, a clustering of the slopes was done in the direction previously selected by means of an agglomerative hierarchical clustering algorithm called AGNES (AGglomerative NESting, see Kaufman and Rousseeuw
Fig. 6 The thickening of the DSM. Each new cell of the DSM was computed using its specific mask. For a new point on a building, its nearest neighbour was chosen using the other points of the building (left). The same procedure was then repeated for the terrain (right). Dark dots cell of the original DSM, positive sign cell of the new $\mathrm{DSM} / /$ mask

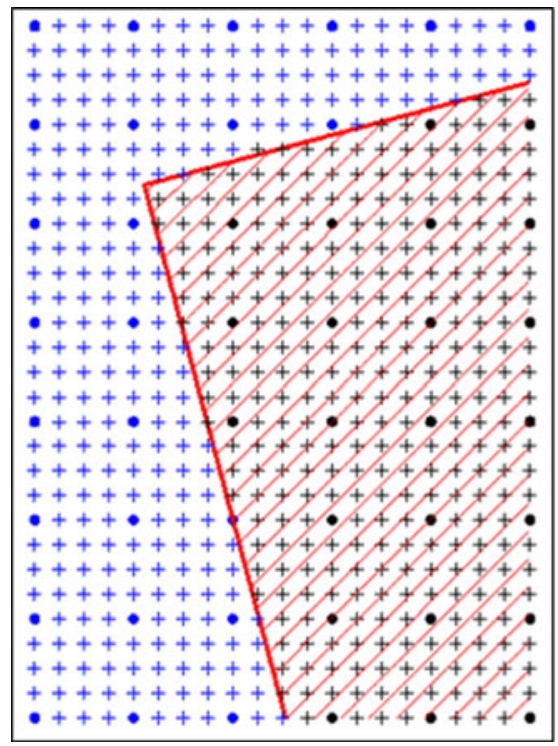

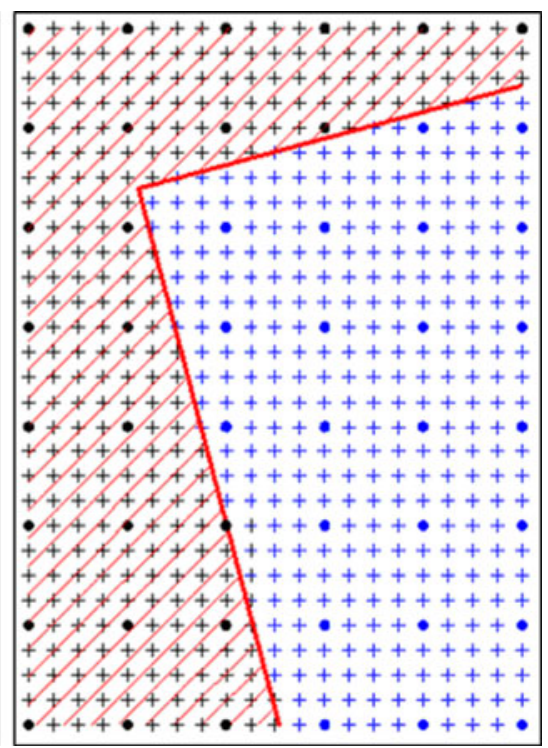




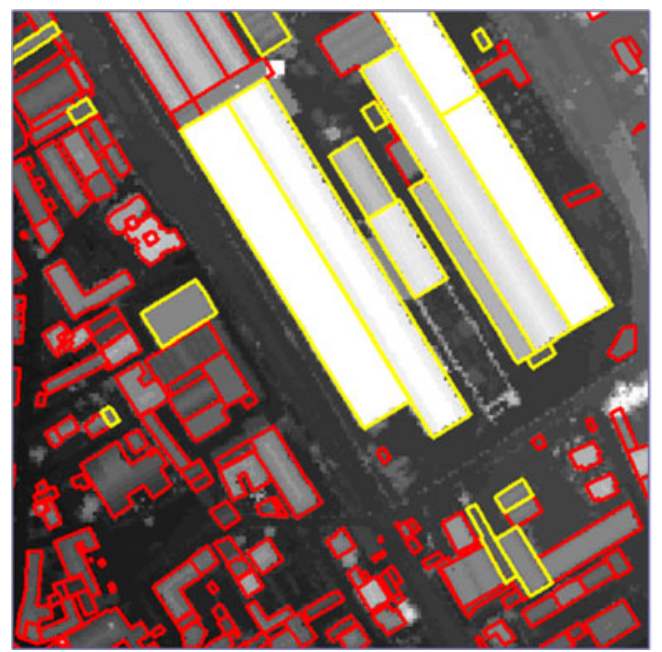

Fig. 7 Buildings modelled with the proposed method (yellow) and with the "thickening method" (red)

1990). This algorithm works in a simple way: in the first pass, all the $n$ instances represent a cluster (each point is a cluster). In the following iterative steps, the closest instances are selected, according to a defined distance measure, and then the number of clusters is updated through merging the selected clusters into a unique cluster. The stopping condition is set according to two joint criteria: the maximum distance criterion and the maximum gradient criterion.

Considering just one aspect class, for a simple gabled roof, we obtained two clusters, one for the points on the principal slope and one for those on the opposite edge; for a roof with several pitches, we obtained as many clusters as triangular sections.

\section{Model reconstruction on a regular grid}

\section{Robust 3D modelling procedure from clusters}

For each building, we were able to obtain corner coordinates from the geodatabase and use the clusters' bounds to divide the different planes. Having those boundary values (duly rotated to be consistent with the used points), we were able to make a regular grid with a raster width of $20 \mathrm{~cm}$ on the $x y$ plane. By means of the parameters estimated with the RANSAC algorithm, we could then build a 3D model of the roofs. In Figs. 3 and 4, examples for a simple gabled roof and a roof with several pitches are illustrated.

In Fig. 5, all the buildings modelled with this new methodology are shown, while the next section presents the strategy used to model all the other buildings in the considered area so that we can obtain a complete model.

Completion of the model

The other buildings located in the area of interest were modelled by adopting the "thickening method", which allows us to model roofs with a complex geometry in an automatic way. The main drawback of the "thickening method" is that it does not provide a regular surface, and this can lead to object edges with unrealistic irregular behaviour (the ribbon-shape effect). However, this procedure was employed to complete the model for the area of interest, also taking into account complex roofs.

The method is based on the thickening of a LiDAR DSM (with a grid size of $2 \mathrm{~m}$ ) to obtain a new DSM with a resolution similar to that of the true orthophoto $(0.2 \mathrm{~m})$. The method requires the $2 \mathrm{D}$ position of the buildings (achievable with a map) and can model buildings with a complex geometry by considering all breaklines given by the 2D map. From this point of view, it represents a valid alternative to the "new" method, and their combined use allows for the creation of a true orthophoto for the whole area.

First, two dense raster masks can be created with the geodatabase, named the building mask (BM) and the terrain mask (TM). The BM contains the buildings, while the TM takes into account all elements that are not buildings (terrain, vegetation, etc.). The thickening is carried out in two steps in order to create two DSMs corresponding to the masks. The procedure is shown in Fig. 6. First, the cells of the DSM are classified as building or terrain with the masks, and, then, the thickening of the building cells can be carried out considering only the points that belong to their specific mask by using the nearest neighbour as an interpolation method. This procedure

Fig. 8 The final model

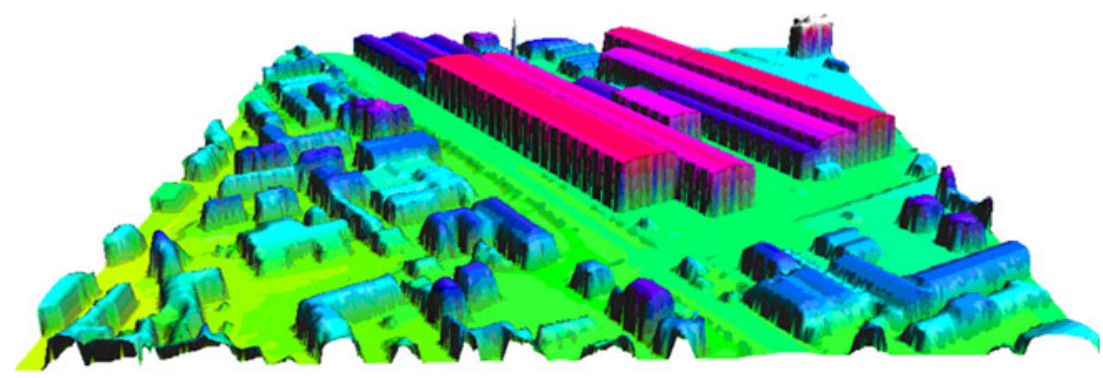




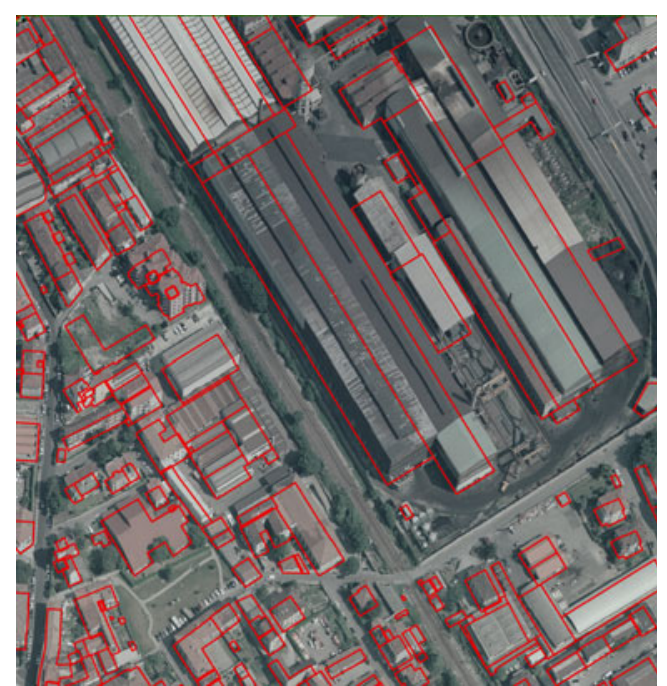

Fig. 9 Orthophoto of the area computed with a $2 \mathrm{~m}$ raster size DTM

allows for a separation between buildings and terrain during the thickening phase; consequently, breaklines can be preserved. The same procedure is then repeated with the terrain mask to obtain a DSM for each element that is not a building. Finally, these two new buildings and terrain DSM can be merged to create a global DSM for the considered area.

In this case, the BM for the buildings already modelled was used to limit the creation of a DSM corresponding to roofs for which the "new" method cannot be used. This choice ensures the creation of true orthophotos in areas with complex roofs.

Figure 7 shows the buildings modelled with the method based on the aspect computation (yellow lines), while the others were modelled with the thickening of the existing DSM (red lines).

The completed model adopted is shown in Fig. 8; the terrain is modelled using a LiDAR DTM resampled at $0.2 \mathrm{~m}$.

\section{True orthophoto generation}

To check the quality of the true orthophoto created using the solution proposed in this paper, we carried out several visual comparisons. In fact, the true orthorectification method here used places each roof into the area defined by the geodatabase. Moreover, sharp edges in the final true orthophotos should be guaranteed by the new DSM. Once this is understood, a visual check of the edges is sufficient to establish the quality of the achieved image. Lastly, we performed a numerical comparison between the DSMs created with the procedure based on the aspect computation and the thickening of the DSM.

\section{Orthophoto with LiDAR DTM}

The original DTM has a raster size of $2 \times 2 \mathrm{~m}$ and was created through the interpolation of last pulses (LiDAR survey) after a preliminary filtering process that removed the "non-terrain" observations.

The orthophoto of the considered area is shown in Fig. 9. Figure 10 shows a detail where one can see the displacement between the geodatabase and the roof position in the image; this displacement is more than $14 \mathrm{~m}$ for a building $23 \mathrm{~m}$ high.

As expected, the LiDAR DTM, even with smaller grid spacing than that commonly used, shows the same distortion in correspondence to attached or detached objects.

\section{True orthophoto with LiDAR DSM}

Another product of a LiDAR survey is the DSM. In this case, the creation of a grid model was based on the interpolation of first pulse data and did not require any preliminary filtering (an exception was made for an outlier removal). The raster size was $2 \times 2 \mathrm{~m}$.
Fig. 10 The displacement between geodatabase and roof position in the orthophoto is clearly visible

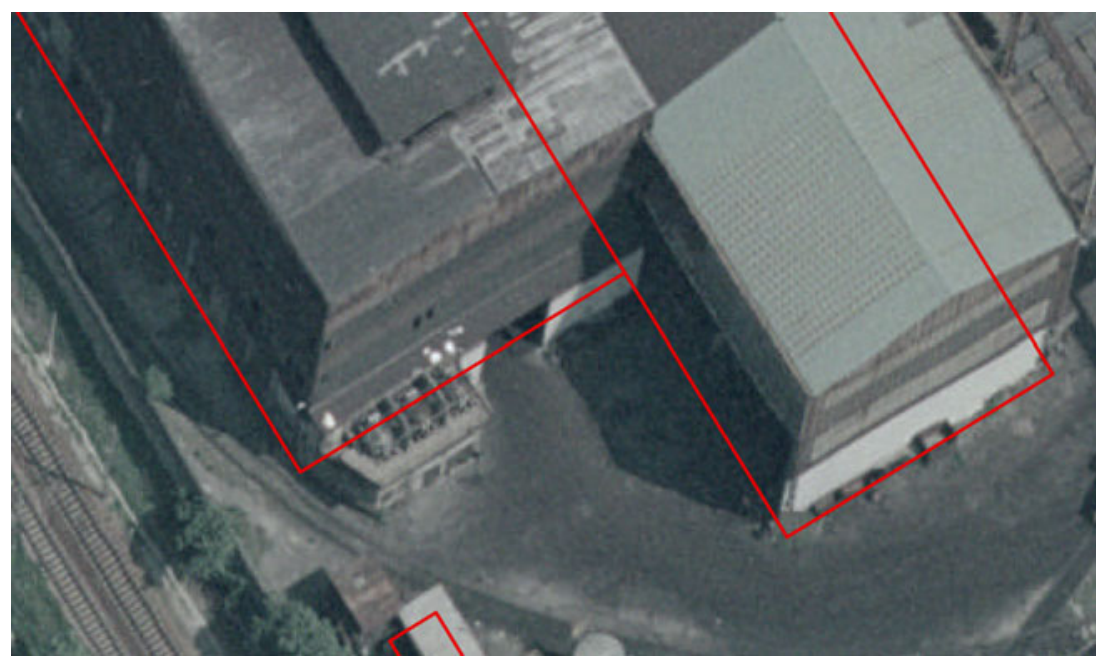


Fig. 11 A detail of the true orthophoto with a $2 \mathrm{~m} \mathrm{DSM}$ showing problems close to the breaklines

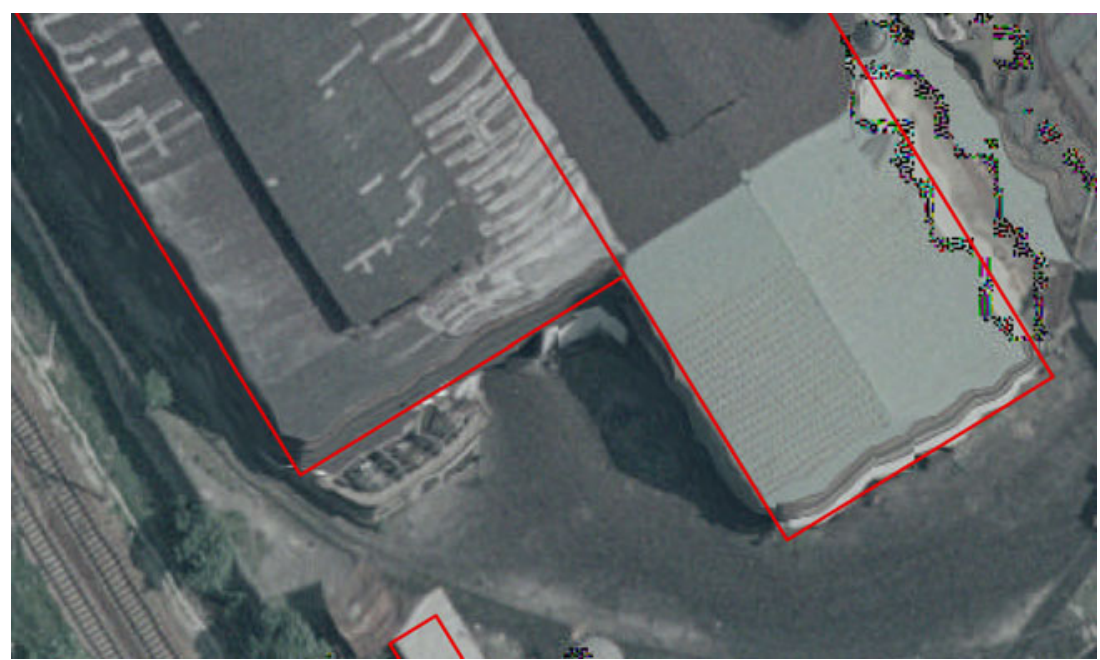

The use of a DSM allowed us to correct the displacement of the roofs, but the different grid size between the DSM $(2 \mathrm{~m})$ and the true orthophoto $(0.2 \mathrm{~m}$ in this case) generated several problems close to the breaklines (in this case roof edges). In fact, if we had simply densified the DSM using a nearest neighbour algorithm or a polynomial interpolation without taking into account points belonging to the roof or the terrain, the final effect in the image would have been an undulating roof edge (Fig. 11). This is the reason that a LiDAR DSM cannot be used directly for the creation of accurate true orthophotos.

True orthophoto with the developed method

The "new" method allows for a refining of the DSM in order to consider the breaklines. With the proposed procedure, one can create a dense grid model that contains information about the position of the building roof with an accuracy of $0.2 \mathrm{~m}$. This is similar to the accuracy of the true orthophoto.

In this case, the building roof in the true orthophoto is forced inside its projection in the geodatabase, and the undulating perimeter effect is significantly limited. Results are shown in Figs. 12 and 13.

As can be seen in Figs. 12 and 13, all buildings are correctly represented, and the border effect is sensibly reduced.

\section{Comparison between new and thickening method}

Orthophotos created with the proposed method were compared with those created with the "thickening method". The main difference between these methods is the possibility of modelling the roof with a more accurate 3D model. In fact, the old method is based on a thickening of a LiDAR
DSM using the nearest neighbour as an interpolation method, which does not provide a regular surface. Representative examples of comparison between the results of the procedures are shown in Figs. 14a and $b$.

Both pictures depict the result after a true orthorectification process using the "thickening" and the "new" method. The result with the "new" method is better than that with the old one, as can be seen in correspondence of the arrows represented in the figures.

The main discrepancies are located close to the breaklines: as expected, the higher the buildings, the greater the differences (see Fig. 15). This is due to the use of original LiDAR observations ("new method") to build a refined model of the roofs instead of simply thickening an existing DSM (as in the "thickening method").

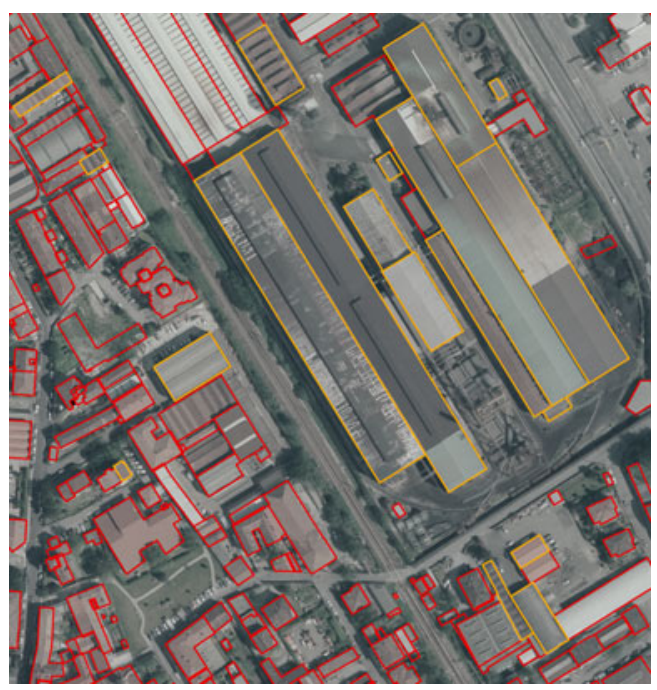

Fig. 12 True orthophoto with the proposed method. Yellow and red lines represent roofs analysed with the method based on the aspect computation and the thickening of the DSM, respectively 
Fig. 13 A detail of the true orthophoto with the proposed model

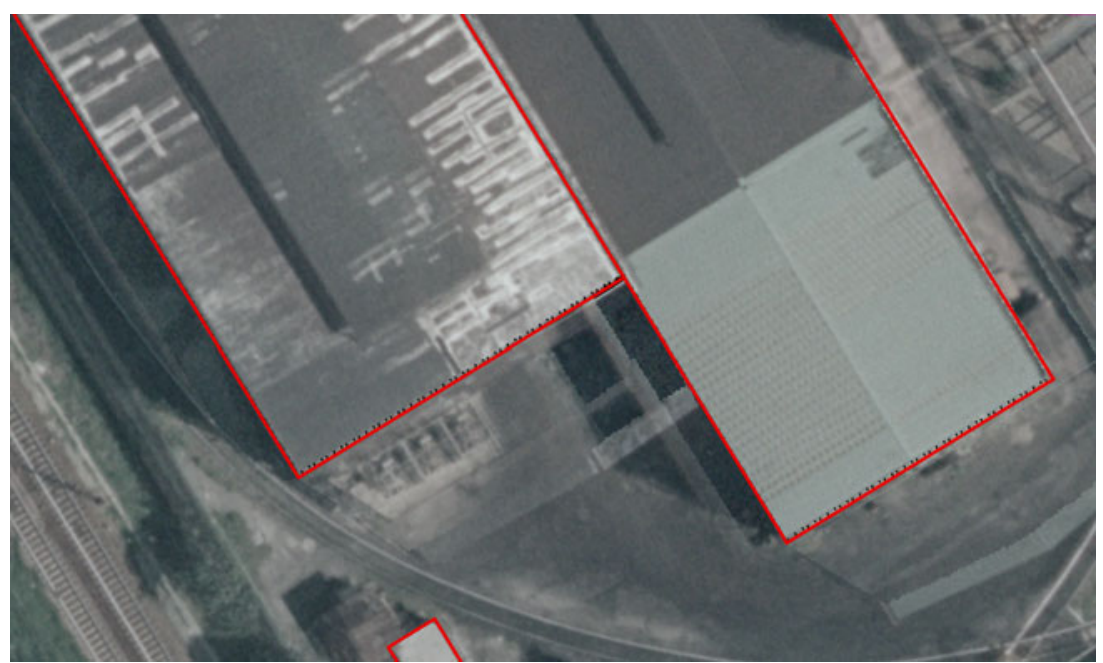

Final considerations and further improvements

In the paper, we faced the problem of the orthorectification of high-resolution aerial images. It is well known that the use of a DTM as a model leads to clearly visible geometric deformations in the resulting image that are more relevant in the case of high buildings and vegetation. Adopting a DSM, the effect is partially
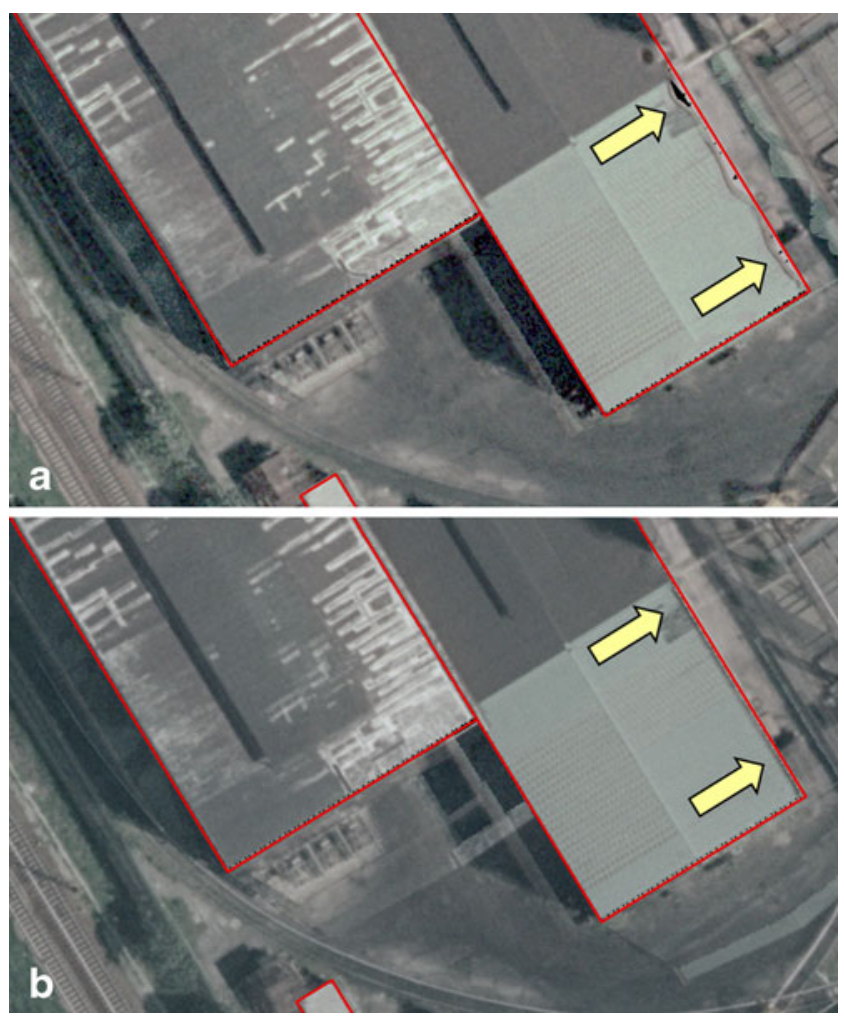

Fig. 14 Comparison between true orthophotos of the same detail with the "thickening method" (a) and the proposed method (b) corrected, but edges show an unrealistic irregular behaviour (the ribbon-shape effect). The DSM densification used to attain a grid spacing comparable with that of the final orthophotos can be obtained through simple algorithms (like the nearest neighbour method), and this procedure partially reduces the unwanted effect. Keeping in mind those considerations, we proceeded in a different way. We developed a fine model of the roofs, limiting ourselves for the moment to a few typologies, with few and several pitches. Thanks to this method, we obtained a further improvement in the geometric correction of the image, especially along breaklines, where the ribbonshape effect is reduced. In addition, the image of a building roof is forced into its $2 \mathrm{D}$ position given by a geodatabase. This means that the spatial accuracy of the true orthophoto is equal to the accuracy of the geodatabase. Further improvements will consist of the extension of the methodology to more complex roofs and vegetation.

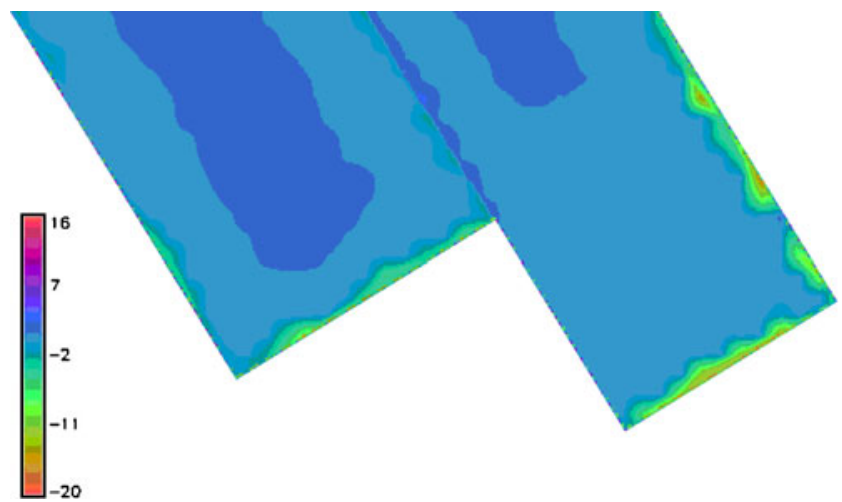

Fig. 15 Raster image of the difference (in metres) between the old and the new $0.2 \mathrm{~m}$ DSMs. The main differences are located close to the roof edges 
Acknowledgments We would like to thank Lecco Municipality, Lombardy Region, and Blom CGR S.p.A, which provided the geodatabase, the LiDAR dataset, and the aerial imagery. This research was partially supported by grants from the Italian Ministry for School, University and Scientific Research (MIUR) in the frame of the project MIUR-COFIN 2007 "Free and open source geoservices as interoperable tools for sharing geographic data through the Internet".

Open Access This article is distributed under the terms of the Creative Commons Attribution Noncommercial License which permits any noncommercial use, distribution, and reproduction in any medium, provided the original author(s) and source are credited.

\section{References}

Amhar F, Jansa J, Ries C (1998) The generation of the true-orthophotos using a 3D building model in conjunction with a conventional DTM. Int Arch Photogrammetry Remote Sens 32(4):16-22

Barazzetti L, Brovelli M A, Scaioni M (2007) Problems related to the generation of true-orthophotos with LiDAR DDSMs. ISPRS Workshop on Laser Scanning 2007 and SilviLaser 2007, pp 20-25

Biason A, Dequal S, Lingua A (2003) A new procedure for the automatic production of true orthophotos. Int Arch Photogrammetry Remote Sens 35:538-543

Brenner C (2000) Towards fully automatic generation of city models. Int Arch Photogrammetry Remote Sens 32:85-92

Braun J (2003) In: Fritsch D, Hobbie D (eds) Aspects on trueorthophoto production. Proc. of Phot. Week '03. Wichmann, Stuttgart, pp 205-214

Dequal S, Lingua A (2004) True orthophoto of the whole town of Turin. Int Arch Photogrammetry Remote Sens 34(5/C15):263-268

De Smith MJ, Goodchild MF, Longley PA (2007) Geospatial analysis - a comprehensive guide to principles. techniques and software tools. Troubador Publishing Ltd, Leicester, pp 267-271

Fischler MA, Bolles RC (1981) Random sample consensus: a paradigm for model fitting with applications to image analysis and automated cartography. Commun ACM 24:381-395

Forlani G, Nardinocchi C, Scaioni M, Zingaretti P (2005) Complete classification of raw LIDAR data and 3D reconstruction of buildings. Pattern Anal Appl 4(8):357-374
Geibel R, Stilla U (2000) Segmentation of laser-altimeter data for building reconstruction: comparison of different procedures. Int Arch Photogrammetry Remote Sens 33(Part B3):326-334

Habib A, Kim E, Kim C (2007) New methodologies for true orthophoto generation. Photogramm Eng Remote Sens 73(1):25-36

Kaufman L, Rousseeuw PJ (1990) Finding groups in data. An introduction to cluster analysis. Wiley, New York, pp 199-252

Kraus K (2007) Photogrammetry. Geometry from images and laser scans. Walter de Gruyter, Berlin

Kuzmin P, Korytnik A, Long O (2004) Polygon-based true orthophoto generation. XXth ISPRS Congress Proceedings, 12-13 July, Istanbul, Turkey, pp 529-531

Maas HG, Vosselman G (1999) Two algorithms for extracting building models from raw laser altimetry data. ISPRS J Photogramm Remote Sens 54:153-163

Rau JY, Chen NY, Chen LC (2002) True orthophoto generation of built-up areas using multi-view images. PE\&RS 68(6):581588

Rottensteiner F (2003) Automatic generation of high-quality building models from LIDAR Data. IEEE Comput Graph Appl 23(6):42-50

Sampath A, Shan J (2008) Building roof segmentation and reconstruction from LiDAR point clouds using clustering techniques. Int Arch Photogrammetry Remote Sens XXXVII(Part B3a):279-284

Schickler W (1998) Operational procedure for automatic true orthophoto generation. IAPRSSIS 32(4):527-532

Ulm K, Poli D (2006) 3D city modelling with Cybercity-Modeler. 1st EARSeL Workshop of the SIG Urban Remote Sensing, 2-3 March, Berlin

Verma V, Kumar R, Hsu S (2006) 3D building detection and modeling from aerial LIDAR data. IEEE Comput Soc Conf Comput Vis Pattern Recognit 2(CVPR'06):2213-2220

Vosselman G (1999) Building reconstruction using planar faces in very high density height data. Int Arch Photogrammetry Remote Sens 32(Part 3-2W5):87-92

Vosselman G, Dijkman S (2001) 3D building model reconstruction from point clouds and ground plans. Int Arch Photogrammetry Remote Sens 34:37-43

Zhou G (2005) Urban large-scale orthoimages standard for national orthophoto program, XXVth IEEE International Geosciences and Remote Sensing Symposium Proceedings, 25-29 July, Seoul, Korea, unpaginated CD-ROM 